\title{
Nonlocal interactions in doped cuprates: correlated motion of Zhang-Rice polarons
}

\author{
L. Hozoi, ${ }^{1,2}$ S. Nishimoto, ${ }^{1}$ G. Kalosakas, ${ }^{1}$ D. B. Bodea,${ }^{1}$ and S. Burdin ${ }^{1}$ \\ ${ }^{1}$ Max-Planck-Institut für Physik Complexer Systeme, Nöthnitzer Str. 38, 01187 Dresden, Germany \\ ${ }^{2}$ Max-Planck-Institut für Festkörperforschung, Heisenbergstrasse 1, 70569 Stuttgart, Germany
}

(Dated: July 14, 2018)

\begin{abstract}
In-plane, inter-carrier correlations in hole doped cuprates are investigated by ab initio multiconfiguration calculations. The dressed carriers display features that are reminiscent of both Zhang-Rice (ZR) $\mathrm{CuO}_{4}$ singlet states and Jahn-Teller polarons. The interaction between these quasiparticles is repulsive. At doping levels that are high enough, the interplay between long-range unscreened Coulomb interactions and long-range phase coherence among the O-ion half-breathing vibrations on the ZR plaquettes may lead to a strong reduction of the effective adiabatic energy barrier associated to each polaronic state. Tunneling effects cannot be neglected for a relatively flat, multi-well energy landscape. We suggest that the coherent, superconducting quantum state is the result of such coherent quantum lattice fluctuations involving the in-plane $\mathrm{O}$ ions. Our findings appear to support models where the superconductivity is related to a lowering of the in-plane kinetic energy.
\end{abstract}

The parent compounds of the high-temperature cuprate superconductors are Mott insulating antiferromagnets. Superconductivity (SC) occurs in these systems upon hole or electron doping. While the magnetic interactions are remarkably strong, there is increasing evidence that many properties cannot be understood without taking into account the lattice degrees of freedom. Experiments that indicate strong electron-phonon (EP) couplings are the inelastic neutron scattering 1, 2], angle-resolved photoemission (PE) 3, 4], X-ray absorption fine structure (XAFS) 5], and electron paramagnetic resonance [6]. Anomalies in the phonon, PE, and XAFS spectra were previously addressed with $t-J$ or Hubbard-like models supplemented with EP interaction terms [7, 8, 9, 10]. Strong and anomalous electron-lattice couplings were also found by $a b$ initio, explicitly correlated calculations 11, 12]. In the case of hole doping 13], multiconfiguration (MC) calculations on clusters of few $\mathrm{CuO}_{6}$ octahedra show that the doped holes enter $\mathrm{O} 2 p_{x}$ and $2 p_{y}$ orbitals that form $\sigma$-bonds with the open-shell $\mathrm{Cu} 3 d_{x^{2}-y^{2}}$ orbitals [1] and give rise to singlet states similar to the kind of configuration proposed by Zhang and Rice (ZR) long time ago 14 . A major deviation from the original ZR picture is that the formation of such a singlet is associated with significant lattice deformations. The most stable configuration corresponds to $\mathrm{Cu}-\mathrm{O}$ distances that are shorter by $5-6 \%$. Most remarkably, O-ion displacements that restore the translational symmetry induce strong charge redistribution. The $2 p$ hole, which for a distorted $\mathrm{CuO}_{4}$ plaquette is equally distributed over the four anions, can be partially transferred onto a single ligand to give an electronic wavefunction (WF) with a dominant contribution from a $\ldots-\mathrm{Cu} d^{9}-\mathrm{O} p^{5}-\mathrm{Cu} d^{9}-$ ... configuration. The ZR-like singlet polaron (ZRP) can hop thus within the $\mathrm{CuO}_{2}$ plane via such $d^{9}-p^{5}-d^{9}$ states through coupling to the oxygen vibrations. The energy barrier associated with the hopping process is few hundreds meV, for an isolated $2 p$ hole [1].

$d^{9}-p^{5}-d^{9}$ entities were postulated to exist in cuprates by several authors [15, 16]. This single-oxygen $2 p$ hole and the adjacent $\mathrm{Cu}$ holes are viewed as a spin- $1 / 2$ quasi- particle state in which the $3 d$ spins are ferromagnetically (FM) polarized, i.e. coupled to a triplet, and the spin on the $\mathrm{O}$ ion is low-spin coupled to it. Interactions that could lead to pairing of these quasiparticles and SC were analyzed in 15, 16 in terms of $p-d$, extended Hubbard Hamiltonians. It was pointed out [16, 17] that such models imply different low-energy physics as compared to the effective single-band model proposed by ZR 14]. Interestingly, the $a b$ initio calculations [11] show that the two concepts, the ZR and the $d^{9}-p^{5}-d^{9}$ configurations, are in fact intimately related. The connecting element is the lattice degree of freedom, more precisely the $\mathrm{Cu}-$ O bond-length fluctuations. Near-degeneracy effects between a quadrisinglet (QS) polaronic state similar to the ZR state and a two-site spin-singlet polaron were studied in the frame of a Holstein-Hubbard model in [18]. Although some details are quite different, the two-site spin-singlet resembles a two-hole $d^{9}-p^{5}$ singlet configuration. It was shown that in the parameter region with near-degeneracy, the effective mass of the QS polaron is sharply reduced. Near-degeneracy plus quantum tunneling effects associated with a rather flat energy landscape are believed to be 18 crucial ingredients in the theory of "bipolaronic" SC.

In this Letter we investigate interactions between two or more spatially separated ZRPs. We find that longrange phase coherence among the O-ion half-breathing vibrations on the $\mathrm{ZR} \mathrm{CuO}_{4}$ plaquettes may lead to a correlated motion of the $2 p$ holes. Several effects come here into play. The local electron-lattice couplings make possible the transfer of the ZR singlet to an adjacent plaquette. The longer-range inter-carrier interactions are responsible, at dopant concentrations that are high enough and in a "dynamic" regime, for a significant lowering of the effective energy barrier seen by each ZR quasiparticle. This is exactly the kind of picture proposed, in a somewhat different context, by Hirsch 19]: single carriers are heavily dressed at very low concentrations by the interaction with the local environment; they partially "undress" and their effective mass decreases at higher concentrations due to collective inter-carrier interactions. 
Our analysis is mainly based on results obtained by $a b$ initio electronic structure methods from traditional quantum chemistry. The calculations are performed on finite clusters. The many-electron WF is constructed as a full configuration-interaction (CI) expansion in the space defined by a limited set of so-called active orbitals. These are chosen as those orbitals that are expected to contribute to degeneracy effects, that is, strong mixing between configurations which have the same, or nearly the same, energy. The rest of the orbitals are either doubly occupied in all configurations or empty. The former set of orbitals is called inactive, the latter is referred to as the virtual orbital set and spans the rest of the orbital space, defined from the basis set used to build the "molecular" orbitals (MOs). This is known as the Complete Active Space (CAS) MC approach 20]. The variational parameters of the WF are both the CI and MO coefficients.

Inter-carrier interactions are studied by doping a 7plaquette $\left[\mathrm{Cu}_{7} \mathrm{O}_{22}\right]$ linear cluster with two holes. The length of the cluster corresponds roughly to the scale of the superconducting coherence length. We used the lattice parameters of the hole doped $\mathrm{La}_{1.85} \mathrm{Sr}_{0.15} \mathrm{CuO}_{4}$ compound [21], with the in-plane lattice constant $a=3.78 \AA$. The 7-plaquette cluster is embedded in an array of formal ionic point charges (PCs) at the experimental lattice positions that reproduce the crystal Madelung potential. The $\mathrm{Cu}^{2+}$ and $\mathrm{La}^{3+}$ nearest neighbors (n.n.) are represented by effective model potentials 22]. Trial calculations were first performed with embedding PCs that correspond to a fully ionic picture of the undoped material, $\mathrm{La}_{2} \mathrm{CuO}_{4}$. In this situation, due to the large mutual repulsion, the CAS Self-Consistent Field (SCF) calculations converge to solutions where the $2 p$ holes are each located on the outermost $\mathrm{CuO}_{4}$ plaquettes. However, at not too small doping other $2 p$ holes exist in the immediate vicinity. In a next step, we decided to take into account the presence of other doped holes by introducing few extra elementary positive charges around the cluster. The results discussed in the following paragraphs were obtained with a PC embedding where eight positive charges were added. The positions of these extra PCs are shown in Fig. 1. The reason behind choosing this type of arrangement is discussed below.

It is widely accepted 23] that charge and magnetic inhomogeneities appear in cuprates at least dynamically. Goodenough 25] argued that at optimal doping $(x \approx 1 / 6)$ and low temperatures, 1/3-doped (fluctuating) stripes are formed on every second chain of plaquettes. In a ZR-like picture, the 1/3-doped stripes could be represented as in Fig. 1(a), for example. The SC is associated in Ref. 25] with the formation of traveling charge-densitywaves [24] along the doped chains. In models with EP coupling, the relevant phonon modes would be the O-ion half-breathing modes with $\mathbf{q}=(1 / 3,0,0)$. Experimental data that appear to support Goodenough's model are the strong anomalies at such wave vectors, $\mathbf{q}=(0.25-$ $0.33,0,0)$ 1, 2]. These anomalies are actually observed in both hole and electron doped systems [2]. Coming

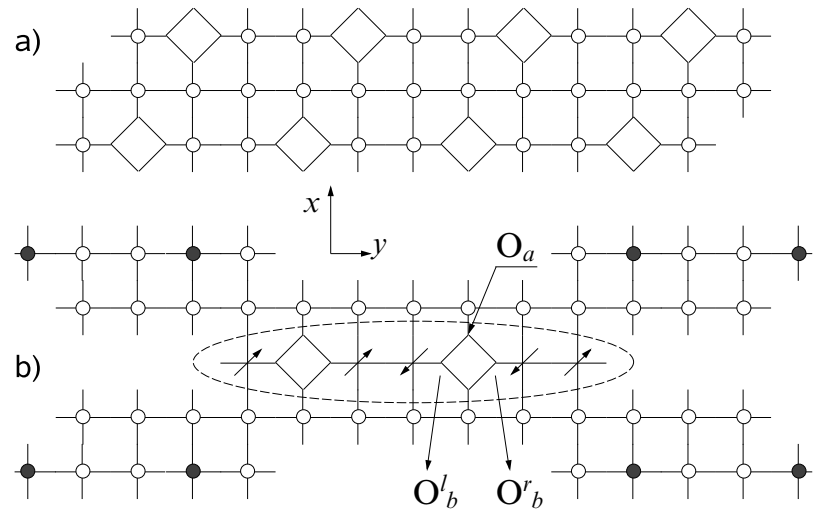

FIG. 1: (a) 1/3-doped stripes at optimal doping in a ZRlike picture, see text. (b) The 7-plaquette cluster employed for the CASSCF calculations and the positions of the extra positive charges (the filled circles) within the PC embedding. These correspond to the situation where every second pair of ZRPs along the $1 / 3$-doped stripes is removed. One of the two symmetry-equivalent lowest-energy configurations is sketched for the "quantum" cluster. Only the $\mathrm{Cu}$ sites are displayed. The squares represent distorted ZR plaquettes.

back to the positive charges added to our embedding, they would correspond to the situation where every second pair of ZRPs in Fig. 1(a) is removed, i.e. $x=1 / 12$ doping. In addition, we retain only the n.n. pairs around the "quantum" cluster. For symmetry reasons, the extra positive charges on the left-hand side are shifted by one plaquette to the right.

With the extra positive charge around the cluster, the two $\mathrm{O}$ holes are pushed from the outermost plaquettes toward the inner region. To study electron-lattice couplings, we first determined the cluster geometry configuration that minimizes the total energy. However, only the positions of those ions are relaxed that have each neighbor represented at the all-electron level. These are the $\mathrm{Cu}$ ions and the intervening ligands $\mathrm{O}_{b}$. Under the assumption of charge segregation into hole-rich and holepoor regions and filamentary conduction 10, 15, 23, 25. the vibrations of these ions should be the most relevant. The CAS SCF calculations were carried out with a minimal active space. That is one orbital per hole, in our case nine electrons in nine orbitals. It was shown 12 that the overall picture does not change when using larger active spaces. Extra orbitals added to the active space turn out to have occupation numbers either very close to 2.00 or to 0 12. Also, when enlarging the active space, it is not obvious how many extra orbitals are required for a balanced description of the different configurations. All calculations were performed with the MOLCAS 6 package [26]. All-electron atomic natural-orbital basis sets from the MOLCAS library were employed, with the following contractions: $\mathrm{Cu} 21 s 15 p 10 d / 5 s 4 p 3 d$, O $14 s 9 p / 4 s 3 p$. As already mentioned, the apical oxygens are not included in the "quantum" cluster, but modeled by formal PCs. 
TABLE I: MPs illustrating the distribution of the O-holes. The lowest-energy configuration corresponds to distorted, ZR plaquettes separated by two other $\mathrm{Cu} 3 d_{x^{2}-y^{2}}^{1}$ ions (1st column). The ZR plaquettes are labeled with indices 1 and 2 . Other notations are as in Fig. 1 . When the $\mathrm{O}_{b}$ ions are shifted back to the middle positions, each hole is partially transferred onto a single ligand (2nd column). In parentheses, the same quantities are shown for a single doped hole, see text.

\begin{tabular}{|c|c|c|}
\hline Configuration & $\begin{array}{l}\text { Dist. } \mathrm{Cu}-\mathrm{O}_{b}, \\
\text { ZRP states }\end{array}$ & Undist. str. \\
\hline $\mathrm{Cu}_{1} 3 d_{x^{2}-y^{2}}$ & $1.03(1.03)$ & $1.09(1.08)$ \\
\hline $\mathrm{O}_{1 b}^{l} 2 p_{y}$ & $1.64(1.62)$ & $1.77(1.76)$ \\
\hline $\mathrm{O}_{1 a} 2 p_{x}(\mathrm{x} 2)$ & $1.61 \quad(1.62)$ & 1.69 (1.68) \\
\hline $\mathrm{O}_{1 b}^{r} 2 p_{y}$ & $1.61(1.62)$ & $1.29 \quad(1.31)$ \\
\hline $\mathrm{Cu}_{2} 3 d_{x^{2}-y^{2}}$ & 1.03 & 1.09 \\
\hline $\mathrm{O}_{2 b}^{l} 2 p_{y}$ & 1.65 & 1.77 \\
\hline $\mathrm{O}_{2 a} 2 p_{x}(\mathrm{x} 2)$ & 1.61 & 1.68 \\
\hline $\mathrm{O}_{2 b}^{r} 2 p_{y}$ & 1.60 & 1.30 \\
\hline Rel. En. (meV) & $0.00 \quad(0.00)$ & $650 \quad(380)$ \\
\hline
\end{tabular}

The CASSCF calculations always converge to states where the $2 p$ holes are separated by more than two lattice constants. However, depending on geometry and the initial guess for the orbitals, these states may have different character. Two symmetry-equivalent minimumenergy geometries were identified. These are configurations where two ZRP states are formed on $\mathrm{CuO}_{4}$ plaquettes that are separated by two other $\mathrm{Cu}$ ions, see Fig. 1(b). The spins on these intervening cations are antiferromagnetically (AFM) coupled. For the ZR plaquettes, the $\mathrm{Cu}-\mathrm{O}_{b}$ distances are shorter by $5 \%$. Mulliken populations (MPs) of the relevant $\mathrm{Cu} 3 d$ and $\mathrm{O} 2 p$ atomic orbitals on these plaquettes are listed in Table I (first column). The composition of the bonding (B) and antibonding $(\mathrm{AB}) d_{x^{2}-y^{2}}-p_{x, y} \sigma$-orbitals is depicted in Fig. 2. Due to correlation effects, the occupation numbers $(\mathrm{ONs})$ of the $\mathrm{AB}$ orbitals are relatively large. Since the charge distribution on the two ZR plaquettes is nearly identical, only the orbitals on one of these plaquettes are shown in the figure.

When the bridging $\mathrm{O}_{b}$ ions are shifted back to the middle positions [11], each of the $2 p$ holes is partially transferred onto a single ligand. This is illustrated in TableI and Fig. 2. The results were obtained by static total energy calculations (adiabatic approximation). It can be seen in Fig. 2 that the doped-hole orbitals have now much weight onto one of the adjacent plaquettes as well. O-ion half-breathing displacements that shorten the $\mathrm{Cu}-\mathrm{O}_{b}$ bonds on these adjacent plaquettes induce propagation of the $\mathrm{ZR}$ singlet(s) along the $\mathrm{Cu}-\mathrm{O}_{b}-\ldots$ chain. We found that the ZR $2 p$ hole is already moved to the other plaquette for distortions of less than $0.5 \%$ from the high-symmetry geometry. The adiabatic energy barrier is in the two-hole cluster $325 \mathrm{meV}$ per hole, see Table I. Separate calculations were performed with a single doped

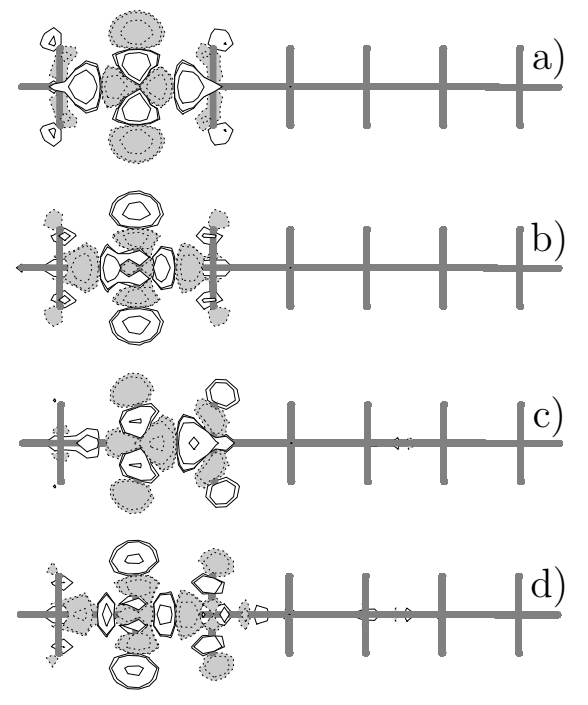

FIG. 2: (a)-(b) B and AB orbitals defining a ZR-like state on a distorted plaquette. Their ONs are 1.86 and 0.14 . (c)-(d) $\mathrm{B}$ and $\mathrm{AB}$ orbitals in the undistorted structure. The ONs are 1.74 and 0.26 . The $2 p$ hole has now the largest weight onto a single "chain" oxygen, see also Table I. The whole 7-plaquette cluster is drawn. The phases of the atomic orbitals are shown with different colors.

hole and no extra positive charge in the PC embedding. The energy barrier is in this case $380 \mathrm{meV}$ [27]. The energy difference between the two situations, $55 \mathrm{meV}$ per hole, is considerable. In the presence of other mobile $2 p$ hole polarons in the $\mathrm{CuO}_{2}$ plane(s), the effect would be further enhanced and should lead to a strong reduction of the polaron effective mass. We arrive thus at Hirsch's paradigm [19, 28]: carriers are not happy being together, but they are happy moving together. This was described as a kinetic-energy-driven mechanism for $\mathrm{SC}$ and a change of color was predicted when entering the superconducting state [19]. That the onset of SC is indeed accompanied by a transfer of spectral weight from the visible region to the infrared range was recently confirmed by Molegraaf et al. [29].

In comparison to the study from Ref.[19] and other model Hamiltonian studies, we are able to identify much more precisely the crucial interactions in the doped plane. The CAS SCF results give also us hints about the nature of the pairing mechanism. It is obvious that this is related to both long-range Coulomb repulsion effects and shorter-range interactions. Our results may be associated with the 4-plaquette correlation bag of Goodenough [25]. The ZR singlet polarons in such a correlation bag are separated by AFM coupled $d_{x^{2}-y^{2}}$ spins that bear some resemblance to the spin-singlet pairs of the resonatingvalence-bond state [30]. $\mathrm{Cu}-\mathrm{O}$ bond-length fluctuations may induce charge transfer along a chain of plaquettes. In the intermediate, undistorted geometry the quasilocalized $2 p$ holes tend to polarize FM the n.n. $3 d$ spins. In other words, the $3 d$ spin on the adjacent plaquette is already "prepared" for the transfer of the $2 p$ hole. Also, 
strong antiferromagnetic correlations exist between the $d^{9}-p^{5}-d^{9}$ units and the "bipolaronic" WF has a complex structure. Our data suggest that two ZR-like quasiparticles could form a 4-plaquette bipolaronic singlet state only above a certain hole concentration. The distribution of "doped" holes displayed in Fig. 1(b) corresponds (at the scale of the figure) to $8-9 \%$ doping, only slightly higher than the concentration(s) where SC actually occurs. We also note that the effects described above, i.e. a lowering of the effective energy barrier due to longerrange interactions, are still obtained when the positions of the extra positive embedding PCs are changed, individually or collectively, by distances as large as one lattice constant.

More investigation is needed for a better understanding of these systems and quantitative estimates. First, a more rigorous treatment of the dynamical electron correlation [20] is required. Secondly, the electronic and lattice degrees of freedom should be described on equal footing, beyond the adiabatic approximation. Another non-trivial task is a self-consistent procedure to determine the (dynamic) in-plane distribution of the charge carriers, perhaps in the spirit of the dynamical meanfield theory [31]. Nevertheless, the purpose of the present study is not to provide highly accurate numbers, but to gain better insight into the very nature of the many-body in-plane interactions. It is our belief that realistic models can only be constructed with the help of this type of first-principles investigations.
To conclude, using a WF-based ab initio approach, we are able to describe microscopic electron-lattice, magnetic, and nonlocal Coulomb interactions characterizing the hole dynamics in (under)doped cuprates. The dressed carriers display features which are reminiscent of both ZR spin-singlet states and Jahn-Teller polarons. We find that at doping levels that are large enough, the adiabatic energy barrier associated to each polaronic state is significantly lower for the synchronized, coherent "hopping" of two ZRPs than in the case of an isolated ZRP. This is due to unscreened inter-carrier Coulomb interactions and requires phase coherence among the Oion half-breathing vibrations. Tunneling effects cannot be neglected for a relatively flat, multi-well energy landscape [10, 18]. Following the discussion from Ref. 18], we suggest that the coherent, superconducting phase is the result of such coherent quantum lattice fluctuations involving the in-plane $\mathrm{O}$ ions. Coherent, collective tunneling effects in the $\mathrm{CuO}_{2}$ planes should lead to extended, "resonant" states [18] and a lowering of the in-plane kinetic energy, see also 19, 28, 32, 33]. Polaronic behavior and local double-well potentials were also found in the electron doped case [12], which suggests some common "global" features for the two types of doping: quantum polarons coupled through spin and longer-range unscreened Coulomb interactions.

We thank I. Eremin and P. Fulde for fruitful discussions.
[1] D. Reznik, L. Pintschovius, M. Ito, S. Iikubo, M. Sato, H. Goka, M. Fujita, K. Yamada, G. D. Gu, and J. M. Tranquada, Nature 440, 1170 (2006).

[2] M. Braden, L. Pintschovius, T. Uefuji, and K. Yamada, Phys. Rev. B 72, 184517 (2005).

[3] A. Lanzara, P. V. Bogdanov, X. J. Zhou, S. A. Kellar, D. L. Feng, E. D. Lu, T. Yoshida, H. Eisaki, A. Fujimori, K. Kishio, J.-I. Shimoyama, T. Noda, S. Uchida, Z. Hussain, and Z.-X. Shen, Nature 412, 510 (2001).

[4] G.-H. Gweon, T. Sasagawa, S. Y. Zhou, J. Graf, H. Takagi, D.-H. Lee, and A. Lanzara, ibid. 430, 187 (2004).

[5] M. Acosta-Alejandro, J. Mustre de León, S. D. Conradson, and A. R. Bishop, J. Supercond.: Incorp. Novel Magn. 15, 355 (2002).

[6] B. I. Kochelaev, J. Sichelschmidt, B. Elschner, W. Lemor, and A. Loidl, Phys. Rev. Lett. 79, 4274 (1997).

[7] M. Capone, G. Sangiovanni, C. Castellani, C. di Castro, and M. Grilli, ibid. 92, 106401 (2004).

[8] O. Rösch and O. Gunnarsson, ibid. 92, 146403 (2004).

[9] S. Ishihara and N. Nagaosa, Phys. Rev. B 69, 144520 (2004).

[10] A. R. Bishop, D. Mihailovic, and J. Mustre de León, J. Phys.: Condens. Matter 15, L169 (2003).

[11] L. Hozoi, S. Nishimoto, and A. Yamasaki, Phys. Rev. B 72, 144510 (2005).

[12] L. Hozoi and S. Nishimoto, cond-mat/0512219 (unpublished).

[13] The reference undoped configuration implies $\mathrm{Cu} 3 d^{9}$ and
O $2 p^{6}$ formal valence states.

[14] F. C. Zhang and T. M. Rice, Phys. Rev. B 37, 3759 (1988).

[15] V. Hizhnyakov and E. Sigmund, Physica C 156, 655 (1988).

[16] V. J. Emery and G. Reiter, Phys. Rev. B 38, 4547 (1988).

[17] V. J. Emery and G. Reiter, ibid. 38, 11938 (1988).

[18] L. Proville and S. Aubry, Eur. Phys. J. B 11, 41 (1999); ibid. 15, 405 (2000).

[19] J. E. Hirsch, Physica C 201, 347 (1992); J. E. Hirsch and F. Marsiglio, Phys. Rev. B 62, 15131 (2000).

[20] T. Helgaker, P. Jørgensen, and J. Olsen, Molecular Electronic-Structure Theory (Wiley, Chichester, 2000).

[21] R. J. Cava, A. Santoro, D. W. Johnson, and W. W. Rhodes, Phys. Rev. B 35, 6716 (1987).

[22] F. Illas, J. Rubio, J. C. Barthelat, Chem. Phys. Lett. 119, 397 (1985); W. R. Wadt and P. J. Hay, J. Chem. Phys. 82, 284 (1985).

[23] S. A. Kivelson, I. P. Bindloss, E. Fradkin, V. Oganesyan, J. M. Tranquada, A. Kapitulnik, and C. Howald, Rev. Mod. Phys. 75, 1201 (2003).

[24] A. M. Gabovich, A. I. Voitenko, J. F. Annett, and M. Ausloos, Supercond. Sci. Technol. 14, R1 (2001).

[25] J. B. Goodenough, J. Phys.: Condens. Matter 15, R257 (2003).

[26] Molcas 6, Department of Theoretical Chemistry, University of Lund, Sweden.

[27] This value is somewhat larger than in [1] because the po- 
larization of the apical-oxygen valence shells is neglected.

[28] J. E. Hirsch, Science 295, 2226 (2002).

[29] H. J. A. Molegraaf, C. Presura, D. van der Marel, P. H. Kes, and M. Li, ibid. 295, 2239 (2002).

[30] P. W. Anderson, ibid. 235, 1196 (1987).

[31] A possibility is also periodic Restricted Open-Shell Hartree-Fock calculations. Nevertheless, a unit cell of twelve plaquettes at optimal doping, see Fig.1, is prohibitively large.

[32] V. J. Emery and S. A. Kivelson, J. Phys. Chem. Solids, 61, 467 (2000).

[33] Th. A. Maier, M. Jarrell, A. Macridin, and C. Slezak, Phys. Rev. Lett. 92, 027005 (2004). 